\title{
Dermatology Life Quality Index among Different Cultures: A Neglected Bias Needs to Be Addressed
}

\author{
Martin Kassir ${ }^{a}$ Ayman Abdelmaksoud ${ }^{b}$ Stephan Grabbe ${ }^{c}$ Mohamad Goldust ${ }^{d}$ \\ aWorldwide Laser Institute, Dallas, TX, USA; ${ }^{\mathrm{b}}$ Mansoura Dermatology, Venerology and Leprology Hospital, \\ Mansoura, Egypt; 'Department of Dermatology, University Medical Center of the Johannes Gutenberg University,

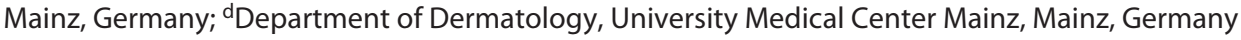

Dear Editor,

Skin is the largest organ of the body, representing a mirror of our physical and psychological condition. Patient self-perception is an important point for disease progression. For more than 20 years, the Dermatology Life Quality Index (DLQI) has been used as a useful benchmark of severity for chronic dermatologic diseases and their management; however, it has several measurement problems. The index is imperfect in capturing the nuances related to ethnographic and cultural peculiarity [1-3].

The psychometric properties of the DLQI have been tested using 55 different language versions in different patient populations. It has been used in many clinical studies in more than 36 dermatologic conditions. Despite the international recommendations for different versions of the DLQI, using the same psychometric assessment tool in various settings without controlling for cultural and linguistic differences is an inherent bias [4-6].

The DLQI has been used differently in each country according to demographic and socioeconomic variables. The impact of socioeconomic status on the DLQI in patients with variable skin conditions, particularly among

karger@karger.com

(c) 2021 S. Karger AG, Basel

www.karger.com/drm

Karger" patients who are female, unemployed, living in rural areas, and having a high income, has been stated $[7,8]$. So, international clinical trial data should not be combined because of cultural differences. This results in false interpretation of scores and variable degrees of response patterns. This neglected bias leads to inaccurate data from merging the clinical trials from different settings and results in fallacious systematic reviews and meta-analyses.

To be an accurate practical measure of patients' psychosocial conditions, the DLQI should be more patient oriented, taking into consideration the variable differences among patients from several ethnic and geographical backgrounds, considering the social diversity among variable regions of the studied population. In addition, in different analyses, for example, gender and age can be taken into consideration. One of these models is the Rasch Analysis, where the differential item function provides this information.

In conclusion, it is important to generate new psychometric questionnaires or modify the existing DLQI, paying more attention to different regions, times, and patients. It is meant to be simple, generalizable, and welldesigned enough as a validated poly-cultural tool. It also 
needs to be vigorously validated and analyzed to make sure that it captures the intended information equally well in various groups of people. This would be a welcome addition to the landscape of dermatology.

\section{Key Message}

It is important to modify the existing Dermatology Life Quality Index based on different regions, times, and patients.

\section{Conflict of Interest Statement}

The authors have no conflicts of interest to disclose.

\section{Funding Sources}

There were no funding sources.

\section{Author Contributions}

Martin Kassir: reviewing the draft, final revision. Ayman Abdelmaksoud: reviewing the draft, final revision. Stephan Grabbe: reviewing the draft, final revision. Mohamad Goldust: writing the draft, final revision. We confirm that the manuscript has been read and approved by all the authors, that the requirements for authorship as stated earlier in this document have been met, and that each author believes that the manuscript represents honest work.

\section{References}

1 Basra MK, Salek MS, Camilleri I, Sturkey R, Finlay AY. Determining the minimal clinically important difference and responsiveness of the Dermatology Life Quality Index (DLQI): further data. Dermatology. 2015; 230(1):27-33.

2 Chernyshov PV. The Evolution of Quality of Life Assessment and Use in Dermatology. Dermatology. 2019;235(3):167-74.

3 Finaly AY, Khan GK. Dermatology Life Quality Index (DLQI) - a simple practical measure for routine clinical use. Clin Exp Dermatol. 1994 May;19(3):210-6.
4 Ali FM, Johns N, Finlay A, Salek MS, Piguet V. Comparison of the paper-based and electronic versions of the Dermatology Life Quality Index (DLQI): evidence of equivalence. $\mathrm{Br}$ J Dermatol. 2017 Nov;177(5):1306-15.

5 Mazzotti E, Barbaranelli C, Picardi A, Abeni $\mathrm{D}$, Pasquini P. Psychometric properties of the Dermatology Life Quality Index (DLQI) in 900 Italian patients with psoriasis. Acta Derm Venereol. 2005;85(5):409-13.

6 Nijsten T, Meads DM, de Korte J, Sampogna F, Gelfand JM, Ongenae K, et al. Cross-cultural inequivalence of dermatology-specific health-related quality of life instruments in psoriasis patients. J Invest Dermatol. 2007; 127:2315-22.

7 Langenbruch A, Radtke MA, Gutknecht M, Augustin M. Does the Dermatology Life Quality Index (DLQI) underestimate the disease-specific burden of psoriasis patients?. J Eur Acad Dermatol Venereol. 2019 Jan;33(1): 123-7.

8 Grob JJ. Why are quality of life instruments not recognized as reference measures in therapeutic trials of chronic skin disorders?. J Invest Dermatol. 2007;127(10):2299-301. 